CLINICAL PRACTICE

\title{
Developing generalism in the South African context
}

\author{
A C Howe, R J Mash, J F M Hugo \\ Professor Amanda Howe is an academic family physician at the University of East Anglia in the UK, Honorary Secretary of the Royal College \\ of General Practitioners, and President-Elect of the World Organization of Family Doctors. Professor Robert Mash is Head of Family Medicine \\ and Primary Care at Stellenbosch University, South Africa, Editor of the African Primary Healthcare and Family Medicine Journal, and chair of \\ the National Education and Training Committee for the South African Academy of Family Physicians. Professor Jannie Hugo is Head of Family \\ Medicine at the University of Pretoria, South Africa and committed to the development of new models of community orientated primary healthcare \\ in the City of Tshwane.
}

Corresponding author: A C Howe (amanda.howe@uea.ac.uk)

The largest impact on the South African burden of disease will be made in community-based and primary healthcare (PHC) settings and not in referral hospitals. Medical generalism is an approach to the delivery of healthcare that routinely applies a broad and holistic perspective to the patient's problems and is a feature of PHC. A multi-professional team of generalists, who share similar values and principles, is needed to make this a reality. Ward-based outreach teams include community health workers and nurses with essential support from doctors. Expert generalists - family physicians - are required to support PHC as well as provide care at the district hospital. All require sufficient training, at scale, with greater collaboration and integration between training programmes. District clinical specialist teams are both an opportunity and a threat. The value of medical generalism needs to be explained, advocated and communicated more actively.

S Afr Med J 2013;103(12):899-900. DOI:10.7196/SAMJ.7509

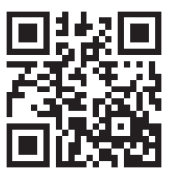

'Medical generalism is an approach to the delivery of healthcare that routinely applies a broad and holistic perspective to the patient's problems. Its principles will be needed wherever and whenever people receive care and advice about their health and wellbeing ...

The ability to practise as a generalist depends on one's training, and on the routine use of skills that helps people to understand and live with their illnesses and disabilities, as well as helping them to get the best out of the healthcare options that are available and appropriate for their needs. [1]

'It involves: (a) seeing the person as a whole and in the context of his or her family and wider social environment; $(b)$ using this perspective as part of the clinical method and therapeutic approach to all clinical encounters; $(c)$ being able to deal with undifferentiated illness and the widest range of patients and conditions; $(d)$ in the context of general practice, taking continuity of responsibility for people's care across many disease episodes and over time; ... (e) coordinating his or her care as needed across organisations within and between health and social care.[1]

\section{Why are generalists needed?}

The World Health Assembly has articulated a need to train and retain adequate numbers of health workers, with the appropriate skill-mix, including primary healthcare nurses, midwives, allied health professionals and family physicians, able to work in a multidisciplinary context, in cooperation with non-professional community health workers to respond effectively to people's health needs..$^{[2]}$

Generalists, working as part of a primary healthcare (PHC) approach, promote equity and better health status. ${ }^{[3]}$ In Africa, the small number of health workers and the difficulty of accessing referral centres, make the need for a competent generalist to manage the burden of multiple concurrent diseases, often in under-resourced settings, more compelling.
Comprehensive care is the hallmark of generalism and means providing a comprehensive service in one setting or from one multi-professional team, and avoiding fragmented disease-specific care. The pursuit of vertical disease-orientated programmes can prevent the development of effective PHC. ${ }^{[4]}$ Generalists also integrate concern for health and disease and can move from cure to palliation while focusing on the whole person.

\section{Models of generalism for the South African context}

South Africa (SA) has set a long-term goal of establishing National Health Insurance (NHI) that would provide equitable and universal coverage for a defined package of healthcare. One of the key pillars of NHI is the re-engineering of PHC, which has at its heart the development of ward-based outreach teams (WBOTs) who will take responsibility for specific groups of households. ${ }^{[5]}$ WBOTs will contribute towards a better understanding of local health needs, inform service priorities and build stronger relationships between service providers and users. The key elements to practise this service are person-centred comprehensive care, collaboration between people and practitioners, and continuity of care. This will be communityorientated primary healthcare (COPC) on a massive scale, and it is estimated that 7000 such teams (community health workers (CHWs) and a nurse, supported by a doctor) are needed. They would provide basic preventive care and health promotion, identify people at risk, support adherence in chronic care, offer home-based care and help integrate care at the community level. Fundamentally, this changes the orientation of the whole system from a reactive facility-based approach to a proactive community-based one.

Several such WBOTs would be supported by a PHC clinic that would be largely nurse-driven, with part-time support from a doctor. In overall support of these WBOTs and clinics, a family physician is required to ensure evidence-based best practice, integrate care, help evaluate and reflect on what is happening, as well as 
mentor and capacitate team members. PHC research will be an important contributor to achieving these goals. ${ }^{\left[{ }^{[6]}\right.}$ Beyond the clinic will be community health centres with multidisciplinary teams, including family physicians and district hospitals. District hospitals, particularly in rural areas, require family physicians with an extended range of skills in hospital care. A new cadre of associate clinicians (mid-level doctors) has been introduced to increase competency at the district hospital. Currently, the need for family physicians in district hospitals seems be emphasised over the need for them in support of PHC. There is a requirement to reclaim the role of the doctor in establishing effective WBOTs and PHC.

Initial assessment of this model in the City of Tshwane shows that COPC can be implemented through WBOTs within the present health system. ${ }^{[7,8]}$ Local, private general practitioners (GPs) are excited about supporting WBOTs and in the next phase GPs will work directly with these teams. In this way, the GP will be able to provide COPC to families in his/her community. In other areas, private GPs are being contracted to support PHC at the clinic level.

Generalist PHC therefore needs to be provided in different, but co-ordinated ways by $\mathrm{CHWs}$, nurses and doctors. Building generalism must include all these role players in an approach that is sensitive to the limitations and potential contribution of each cadre and which builds effective teamwork. Depth of training varies greatly from a few days or weeks for CHWs, to a year for clinical nurse practitioners, and four years for family physicians.

\section{Implications for training}

'Medical generalists are defined by their training, setting, scope of practice, and the retention of a broad skill set and ethos ... It sees the ethos of a generalist as a specific professional orientation that makes different demands from those on a specialist, and needs different training and self-discipline to be effective. ${ }^{[9]}$

The current training of CHWs, nurses and doctors does not necessarily build a shared understanding of generalism. The training of CHWs is still being conceptualised, but may well be too brief for the role that is envisaged. As first contact care is largely offered by nurses, it is vital that they develop the generalist skill set. ${ }^{[10]}$ Greater collaboration between the institutions involved in such training is essential.

Expertise in medical generalism also varies among doctors. Most private GPs and public medical officers have not received any postgraduate training for the roles they occupy, and rely on continuing professional development to extend or refresh their skills. Family physicians who have received a specialist four-year training to be expert generalists are few in number. The challenge therefore for family medicine training programmes is to maximise the number of future family physicians and to re-orientate and 'up skill' the existing doctors for their new roles in a re-engineered PHC. Training programmes must go to a scale; one suggestion is to develop a shorter national diploma-level course, aimed at existing doctors. In the long term we should work towards a scenario where all generalist doctors are family physicians with postgraduate training and recognition as such. The World Organization of Family Doctors has published some possible standards which provide useful direction. ${ }^{[11]}$

If more generalist doctors are needed, we need to ensure that students in medical school become inspired about 'generalism. The modern medical curriculum requires greater emphasis on community-based learning and patient centredness. ${ }^{[12]}$ Doctors and nurses must want to work in the challenging environment of the generalist, and to respect this as an important field of practice.
Investment is needed in the infrastructure and resources required for the training of generalists in community-based, PHC and district settings. District health services should see these training programmes as an opportunity to strengthen healthcare and not as a threat to service delivery.

\section{Threats and opportunities}

Generalists need to build alliances to educate others about what medical generalists do and why it matters. National bodies such as the South African Academy of Family Physicians (SAAFP) can fulfil a crucial role in this regard.

The recent creation of District Clinical Specialist Teams in SA is both a threat and an opportunity for medical generalism. On the one hand, these teams are dedicated to improving maternal and child care, by bringing specialist skills into the district. Teams will provide clinical outreach, mentoring, training and clinical governance activities. For specialists trained in referral hospitals, however, these roles are very different and they are not used to being located within the district health system. There is a danger that they may bring inappropriate assumptions regarding vertical diseaseorientated programmes and hospital-based care into a generalist PHC environment. The placement of specialist family physicians in these teams is a recognition of their important role, but by regarding them as the equivalent of hospital-based specialist, and asking them to only focus on maternal and child health, their generalist nature is subverted and their place as expert generalists employed throughout the district health services may be lost.

Conflict of interest. The SAAFP sponsored Prof. A C Howe's attendance at the National Family Practitioner's Conference to give a plenary address on the topic of generalism. Prof. A C Howe is also an Officer of the Royal College of GPs, and President Elect of the World Organisation of Family Doctors, but the views expressed here are her own.

Acknowledgements. This article is based in part on keynote addresses given by Proff A C Howe and J F M Hugo at the 16th National Family Practitioners Conference in Cape Town, SA, in May 2013 themed 'Reclaiming Generalism.'

1. Royal College of General Practitioners. Independent Commission on Generalism. Guiding patients through medical complexity. London: RCGP, 2011. http://www.rcgp.org.uk/ (accessed 14 August 2013).

2. World Health Assembly. Resolution 62.12: Primary Health Care, Including Health Systems. Geneva: 62nd World Health Assembly, 18 - 22 May 2009.

World Health Organization. The World Health Report 2008: Primary Health Care - Now more than ever. Geneva: WHO, 2008.

4. de Maeseneer I, van Weel C, Egilman D, Mfenyana K, Kaufman A, Sewankambo N. Strengthening primary care: Addressing the disparity between vertical and horizontal investment. Br J Gen Pract 2008:58(546):3-4. [http://dx.doi.org/10.3399/bigp08X263721]

2008;58(546):3-4. [http://dx.doi.org/10.3399/bjgp08X263721]

5. National Dep
NDoH, 2011.

6. Beasley JW, Starfield B, van Weel C, Rosser WW, Haq CL. Global health and primary care research. J Am Board Fam Med 2007;20(6):518-526. [http://dx.doi.org/10.3122/jabfm.2007.06.070172]

7. Bam N, Marcus T, Hugo J, Kinkel H. Conceptualizing community oriented primary care (COPC) - the Tshwane, South Africa, health post model. Afr J Prm Health Care Fam Med 2013;5(1):3 pages. [http:// dx.doi.org/10.4102/phcfm.v5i1.423]

8. Kinkel H, Marcus T, Memon S, Bam N, Hugo J. Community oriented primary care in Tshwane District, South Africa: Assessing the first phase of implementation. Afr J Prm Health Care Fam Med 2012;5(1):477. [http://dx.doi.org/10.4102/phcfm.v5i1.477]

9. Howe A. Medical Generalism: Why Expertise in Whole Person Medicine Matters. London: RCGP 2012. http://www rcgporg uk/policy/rcgp-policy-areas/ /media/Files/Policy/A-Z-policy/Medical2012. http://www.rcgp.org.uk/policy/rcgp-policy-areas/ /media/Files/Policy/A-Z-policy/Medical-

Generalism-Why_expertise_in_whole_person_medicine_matters.ashx (accessed 5 November 2013). 2012;7(3):e32358. [http://dx.doi.org/10.1371/journal.pone.0032358]
a

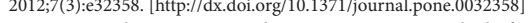

. WONCA Working Party on Education. WONCA Standards for Postgraduate Family Medicine Education. Bangkok: WONCA, 2013. http://www.globalfamilydoctor.com/site/DefaultSite/filesystem/ documents/Groups/Education/WONCA\%20ME\%20stds.pdf (accessed 5 November 2013).

12. Howe A, Campion P, Searle J, Smith H. New perspectives - approaches to medical education at four new UK medical schools. BMJ 2004;329(7461):327-321. [http://dx.doi.org/10.1136/bmj.329.7461.327]

Accepted 8 October 2013. 